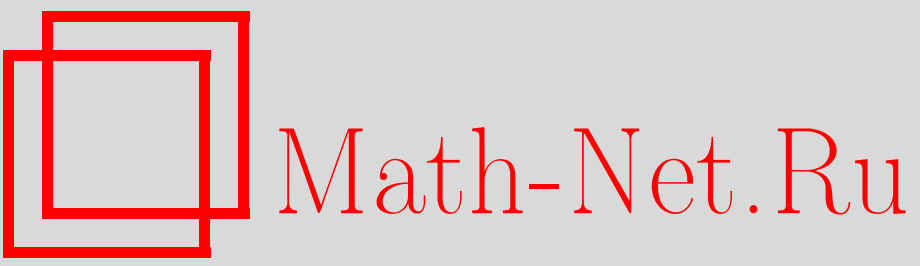

Р. М. Абсава, О предельном распределении квадратического уклонения широкого класса оценок функциональных характеристик закона распределения наблюдений, УМH, 2001, том 56, выпуск 5, 175-176

DOI: https://doi.org/10.4213/rm440

Использование Общероссийского математического портала Math-Net.Ru подразумевает, что вы прочитали и согласны с пользовательским соглашением

http: //www. mathnet.ru/rus/agreement

Параметры загрузки:

IP : 3.85 .183 .62

26 апреля 2023 г., 17:51:51 


\title{
О ПРЕДЕЛЬНОМ РАСПРЕДЕЛЕНИИ КВАДРАТИЧЕСКОГО УКЛОНЕНИЯ ШИРОКОГО КЛАССА ОЦЕНОК ФУНКЦИОНАЛЬНЫХ ХАРАКТЕРИСТИК ЗАКОНА РАСПРЕДЕЛЕНИЯ НАБЛЮДЕНИЙ
}

\author{
P. М. Авсава
}

Пусть $X, X_{1}, \ldots, X_{n}$ - последовательность независимшіх одинаково распределенных случайных величин, принимающих значения в $\mathbb{R}^{p}$, с общей функцией распределения $F(x)$ и плотностью распределения $f(x)$. Допустим, что $g(x), x \in \mathbb{R}^{p},-$ некоторая функциональная характеристика распределения случайной величины $X$, относительно которой предположим, что она принадлежит достаточно широкому множеству функций. По наблюдениям $X_{1}, \ldots, X_{n}$ требуется построить последовательность случайных функций $g_{n}(x)=g_{n}\left(x, X_{1}, \ldots, X_{n}\right)$, которые могут быть приближением $g(x)$.

Приведем некоторые специальные примеры оцениваемых функциональных характеристик:

$D$ - плотность распределения: $g(x)=f(x)$,

$H$ - функция риска: $g(x)=\frac{f(x)}{1-F(x)}$,

$R$ - функция регрессии: $g(x)=m(x)=\mathrm{E}(Y \mid \bar{X}=x)$,

$$
\bar{X}=\left(X^{(1)}, \ldots, X^{(p)}\right), \quad X=(\bar{X}, Y) .
$$

Рассмотрим непараметрические оценки кривых общего вида:

$$
g_{n}(x)=\sum_{i=1}^{n} \delta_{0 \lambda}\left(x, X_{i}\right) / \sum_{i=1}^{n} \delta_{1 \lambda}\left(x, X_{i}\right),
$$

где $\delta_{i \lambda}(x, y), i=0,1,-$ измеримые по Борелю функции в $\mathbb{R}^{p} \times \mathbb{R}^{p}$, а $\lambda=\lambda(n)$ - последовательность положительных чисел, сходящихся к бесконечности, но удовлетворяющих условию $\lambda(n)=o(n)$ при $n \rightarrow \infty$.

Цель работы - изучить предельное распределение квадратического уклонения оценки (1).

Введем обозначения $(r(x)$ - некоторая весовая функция):

$$
\begin{gathered}
\delta_{\lambda}\left(x, X_{i}\right)=\delta_{0 \lambda}\left(x, X_{i}\right)-\delta_{1 \lambda}\left(x, X_{i}\right) g(x), \quad \widehat{g}_{n}(x)=\frac{1}{n} \sum_{i=1}^{n} \delta_{\lambda}\left(x, X_{i}\right), \\
V_{n}=n \int\left(\widehat{g}_{n}(x)-\mathrm{E} \widehat{g}_{n}(x)\right)^{2} r(x) d x, \quad \Delta_{n}=\mathrm{E} V_{n}, \quad \alpha_{\lambda}(x, y)=\delta_{\lambda}(x, y)-\mathrm{E} \delta_{\lambda}\left(x, X_{1}\right), \\
\delta_{i j}(u, v, \lambda)=\int \delta_{i \lambda}(x, u) \delta_{j \lambda}(x, v) g^{i+j}(x) r(x) d x, \quad i, j=0,1, \\
\sigma_{n}^{2}=2 \iint\left(\mathrm{E} \alpha_{\lambda}\left(u_{1}, X_{1}\right) \alpha_{\lambda}\left(u_{2}, X_{2}\right)\right)^{2} r\left(u_{1}\right) r\left(u_{2}\right) d u_{1} d u_{2}, \\
L_{n}=\mathrm{E}\left[\iint \alpha_{\lambda}\left(u_{1}, X_{1}\right) \alpha_{\lambda}\left(u_{2}, X_{2}\right) \mathrm{E} \alpha_{\lambda}\left(u_{1}, X_{1}\right) \alpha_{\lambda}\left(u_{2}, X_{2}\right) r\left(u_{1}\right) r\left(u_{2}\right) d u_{1} d u_{2}\right]^{2}
\end{gathered}
$$

ТеОРема 1. Пусть выполнены условия:

$$
\begin{aligned}
& 1^{\circ} \mathrm{E}\left(\int \delta_{0 \lambda}^{2}\left(x, X_{1}\right) r(x) d x\right)^{\gamma} \leqslant c_{1} \lambda^{\alpha \gamma}, \quad \mathrm{E}\left(\int \delta_{1 \lambda}^{2}\left(x, X_{1}\right) g^{2}(x) r(x) d x\right)^{\gamma} \leqslant c_{2} \lambda^{\gamma \alpha}, \\
& \alpha>0, \gamma=1,2, \\
& 2^{\circ} \mathrm{E}\left(\delta_{i j}^{s}\left(X_{1}, X_{2}, \lambda\right)\right) \leqslant c_{3} \lambda^{(s-\beta) \alpha}, \quad s=2,4, \alpha>0,0<\beta \leqslant 1, i, j=0,1, \\
& 3^{\circ} \sigma_{n} \rightarrow \infty u L_{n}=o\left(\sigma_{n}^{4}\right) \text { npu } n \rightarrow \infty, \\
& 4^{\circ} n^{-1} \sigma_{n}^{-4} \lambda^{(4-\beta) \alpha} \rightarrow 0 \text { nрu } n \rightarrow \infty .
\end{aligned}
$$


Тогда

$$
\sigma_{n}^{-1}\left(V_{n}-\Delta_{n}\right) \stackrel{d}{\rightarrow} N(0,1),
$$

где d обозначает сходимость по распределению, а $N(0,1)$ - случайную величину, имеющ,ую нормальное распределение со средним 0 и дисперсией 1.

Для доказательства теоремы 1 в основном используется центральная предельная теорема для семимартингалов Р.Ш. Липцера и А.Н. Ширяева [1].

Основная теорема 1 дает возможность для известных оценок $D$-Розенблатта-Парзена, $D$-Ченцова, $R$-Надарая-Ватсона и $H$-Ватсона-Лидбеттера единым образом получить предельные распределения квадратического уклонения в уточненном и обобщенном виде по сравнению с имевшимися ранее [2]-[6]. Для примера рассмотрим ядерные оценки $D$-Розенблатта-Парзена.

Пусть $\delta_{0 \lambda}\left(x, X_{i}\right)=\lambda^{p} K\left(\lambda\left(x-X_{i}\right)\right)$ и $\delta_{1 \lambda}\left(x, X_{i}\right)=1$, тогда из $(1)$ получим оценку Розенблатта-Парзена для плотности распределения, т.е. ядерную оценку:

$$
g_{n}(x)=\frac{\lambda^{p}}{n} \sum_{i=1}^{n} K\left(\lambda\left(x-X_{i}\right)\right) .
$$

Назовем функцию $K(x), x \in \mathbb{R}^{p}$, функцией класса $H_{\nu}(\nu \geqslant 2)$, если

1. $\int K(x) d x=1, \quad 2 . \sup _{x \in \mathbb{R}^{p}}|K(x)|<\infty, \quad 3 . \int x_{1}^{i_{1}} \cdots x_{p}^{i_{p}} K(x) d x=0$, если $\sum_{j=1}^{p} i_{j}<\nu$, 4. $\int\left|x_{1}^{i_{1}} \cdots x_{p}^{i_{p}}\right||K(x)| d x<\infty$, если $\sum_{j=1}^{p} i_{j}=\nu, \quad 5 . r(x)=r_{1}\left(x_{1}\right) \cdots r_{p}\left(x_{p}\right), r_{j}\left(x_{j}\right), x_{j} \in \mathbb{R}^{1}$, - кусочно-непрерьвные и ограниченные функции.

ТеОРема 2. Пусть $K(x) \in H_{\nu}(\nu \geqslant 2)$ и $f(x)$ ограничена и имеет ограниченные частные производные до порядка $\nu$ включительно.

a) $\operatorname{Ecлu~} n^{-1} \lambda^{p} \rightarrow 0 u n \lambda^{-p-2 \nu} \rightarrow 0$, mo

$$
\lambda^{p / 2} \sigma^{-1}\left(T_{n}-\theta_{n}^{(1)}-\theta_{n}^{(2)}\right) \stackrel{d}{\rightarrow} N(0,1) .
$$

б) $E$ сли $n^{-1} \lambda^{p} \rightarrow 0$ u $n \lambda^{-p / 2-2 \nu} \rightarrow 0$, mo $\lambda^{p / 2} \sigma^{-1}\left(T_{n}-\theta_{n}^{(2)}\right) \stackrel{d}{\rightarrow} N(0,1)$.

в) Если к условиям, наложенным на $K(x)$, добавить $K(-x)=K(x) u n^{-1} \lambda^{p} \rightarrow 0$, $n \lambda^{-p / 2-2 \nu} \rightarrow 0,1 \leqslant p \leqslant 3$, mo

$$
\lambda^{p / 2} \sigma^{-1}\left(T_{n}-\int f(x) r(x) d x \int K^{2}(x) d x\right) \stackrel{d}{\rightarrow} N(0,1) .
$$

Здесь $T_{n}=\frac{n}{\lambda^{p}} \int\left(g_{n}(x)-f(x)\right)^{2} r(x) d x, \quad \theta_{n}^{(1)}=\frac{n}{\lambda^{p}} \int\left(\mathrm{E} g_{n}(x)-f(x)\right)^{2} r(x) d x, \theta_{n}^{(2)}=$ $\lambda^{p} \iint K^{2}(\lambda(x-u)) f(u) r(u) d u d x, \sigma^{2}=2 \int f^{2}(x) r^{2}(x) d x \int K_{0}^{2}(x) d x, K_{0}=K * K$.

\section{СПИСОК ЛИТЕРАТУРЫ}

[1] Р.Ш. Липцер, А.Н. Ширяев // Теория вероятн. и ее примен. 1980. Т. 25. № 4. C. 683-703. [2] P. Bickel, M. Rosenblatt // Ann. Statist. 1973. V. 1. №6. P. 1071-1095. [3] Э.А. Надарая // Теория вероятн. и ее примен. 1976. Т. 21. №4. С. 864-871. [4] Э. А. Надарая // Сообщ. АН ГССР. 1984. Т. 113. № 2. С. 253-256. [5] Э. А. Надарая // Сообщ. АН ГССР. 1974. Т. 74. № 1. С. 33-36. [6] P. Holl // J. Multivariate Anal. 1974. V. 14. P. 1-16. 